\title{
Longitudinal Changes in Prefrontal Cortex Activation Underlie Declines in Adolescent Risk Taking
}

\author{
Yang Qu, ${ }^{1}$ Adriana Galvan, ${ }^{3,4}$ Andrew J. Fuligni, ${ }^{3,5}$ Matthew D. Lieberman, ${ }^{3,5}$ and Eva H. Telzer ${ }^{1,2}$ \\ ${ }^{1}$ Department of Psychology, ${ }^{2}$ Beckman Institute for Advanced Science and Technology, University of Illinois, Urbana-Champaign, Illinois 61820, and \\ ${ }^{3}$ Department of Psychology, ${ }^{4}$ Brain Research Institute, ${ }^{5}$ Department of Psychiatry and Biobehavioral Sciences, University of California, Los Angeles, Los \\ Angeles, California 90095
}

Adolescence is a critical developmental phase during which risk-taking behaviors increase across a variety of species, raising the importance of understanding how brain changes contribute to such behaviors. While the prefrontal cortex is thought to influence adolescent risk taking, the specific ways in which it functions are unclear. Using longitudinal functional magnetic resonance imaging in human adolescents, we found that ventrolateral prefrontal cortex (VLPFC) activation decreased during an experimental risk-taking task over time, with greater declines in VLPFC associated with greater declines in self-reported risky behavior. Furthermore, greater decreases in functional coupling between the medial prefrontal cortex (MPFC) and ventral striatum over time were associated with decreases in self-reported risky behavior. Thus, disparate roles of the VLPFC and MPFC modulate longitudinal declines in adolescent risk taking.

Key words: adolescence; fMRI; prefrontal cortex; risk taking

\section{Significance Statement}

Adolescence is a developmental period marked by steep increases in risk-taking behavior coupled with dramatic brain changes. Although theories propose that the prefrontal cortex (PFC) may influence adolescent risk taking, the specific ways in which it functions remain unclear. We report the first longitudinal functional magnetic resonance imaging study to examine how neural activation during risk taking changes over time and contributes to adolescents' real-life risk-taking behavior. We find that longitudinal declines in activation of the ventrolateral PFC are linked to declines in adolescent risk taking, whereas the medial PFC influences adolescent risk taking via its functional neural coupling with reward-related regions. This is the first study to identify the mechanism by which different regions of the PFC disparately contribute to declines in risk taking.

\section{Introduction}

In humans, rodents, and primates, adolescent youth exhibit an increase in risk taking (Spear, 2000; Laviola et al., 2003). Despite broad interest in the neural mechanisms of adolescent risk taking, there are few longitudinal studies demonstrating the brain mechanisms underlying changes in adolescent risk taking over time (Pfeifer et al., 2011; van Duijvenvoorde et al., 2014). Several theoretical models propose that subcortical brain regions involved in affective processing and reward sensitivity [e.g., ventral stria-

Received April 21, 2015; revised June 30, 2015; accepted July 3, 2015.

Author contributions: A.G., A.J.F., M.L., and E.H.T. designed research; A.G., A.J.F., M.L., and E.H.T. performed research; Y.Q. and E.H.T. analyzed data; Y.Q., A.G., A.J.F., M.L., and E.H.T. wrote the paper.

This work was supported by the National Institute of Child Health and Human Development (R01HD057164-S and R01HD057164; A.J.F.) and the National Science Foundation (SES 1023293; A.J.F. and E.H.T.).

The authors declare no competing financial interests.

Correspondence should be addressed to either of the following: Eva H. Telzer, 603 East Daniel St., Champaign, IL 61820, E-mail: ehtelzer@illinois.edu; or Yang Qu, 603 East Daniel St., Champaign, IL 61820, E-mail: yangqu3@illinois.edu.

DOI:10.1523/JNEUROSCI.1553-15.2015

Copyright $\odot 2015$ the authors $\quad 0270-6474 / 15 / 3511308-07 \$ 15.00 / 0$ tum (VS)] peak in functional reactivity during mid-adolescence, whereas cortical brain regions supporting cognitive control [e.g., the prefrontal cortex (PFC)] undergo more protracted functional development into early adulthood (Ernst et al., 2006; Casey et al., 2008; Steinberg, 2008). However, in the absence of direct longitudinal evidence, the function of the PFC during risk taking has been debated. Among cross-sectional research examining the role of the PFC in cognitive control across various tasks, both age-related increases and decreases in PFC activity between childhood and adulthood have been found (Bunge et al., 2002; Booth et al., 2003; Durston et al., 2006; Marsh et al., 2006; Rubia et al., 2007; Velanova et al., 2009; Crone and Dahl, 2012). To reconcile these disparate findings, longitudinal studies can provide a better understanding of the PFC's role in risk taking over time. Moreover, multiple subareas of the PFC [e.g., ventrolateral PFC (VLPFC) and medial PFC (MPFC)] may function in different ways during risk taking. For example, the VLPFC has been implicated in cognitive regulation, and may directly affect adolescent risk taking by serving as the neural brake system and stopping the motor response of risky behavior (Wessel et al., 2013). In con- 
trast, the MPFC may play an indirect role in risk taking by regulating subcortical areas and thereby decreasing the affective nature of risk taking. Cross-sectional work in human and rodent youth has shown that from childhood to adolescence, individuals demonstrate a developmental switch from positive to negative coupling between the MPFC and subcortical regions with negative coupling related to better emotional regulation and adjustment (Perlman and Pelphrey, 2011; Gee et al., 2013).

We examined the neurodevelopmental changes underlying adolescent risk taking in three ways. First, we investigated changes in neural reactivity in cognitive control and affective systems to determine whether adolescents demonstrate mean level increases, decreases, or stability in neural reactivity during risk taking across time. Second, we examined individual differences in such changes and tested whether neurodevelopmental changes covary with changes in self-reported risk taking over time. Examining individual variability in neurodevelopmental change will help clarify which neural patterns are associated with increases or decreases in real-life risk-taking behavior. Third, neurodevelopmental changes may not only manifest through changes in reactivity in the PFC and subcortical systems, but also through how these neural systems communicate with each other over time. By examining the coordination of cognitive and affective systems contributing to risk taking, we can determine whether or not they function together in increasingly mature ways (i.e., greater negative coupling; Gee et al., 2013) over time to regulate risk-taking behavior.

\section{Materials and Methods}

\section{Participants}

Twenty-four adolescents completed two fMRI scans, $\sim 1.5$ years apart, a developmental window characterized by significant changes in brain function (van den Bulk et al., 2013). Two participants were excluded from analyses due to excessive head movement (i.e., $>2.5 \mathrm{~mm}$ ). Our final sample comprised 22 adolescents ( 14 females; T1 mean age $=15.77 \pm$ 0.61 years; range $=15.35-17.14$ years; $\mathrm{T} 2$ mean age $=17.14 \pm 0.71$ years; range $=16.44-18.43$ years). Participants completed written consent and assent in accordance with University of California, Los Angeles's Institutional Review Board. Participants were not currently taking any medications and did not report being diagnosed with any mood disorders.

\section{Behavioral measures}

At both $\mathrm{T} 1$ and $\mathrm{T} 2$, adolescents reported on their engagement in risktaking behaviors (e.g., smoking, alcohol and drug use, stealing) using the rule-breaking subscale of the Youth Self-Report (YSR; Achenbach, 1991). The YSR is a well validated measure for adolescents' problem behaviors. The rule-breaking subscale captures a wide range of adolescent risky behavior, such as smoking, alcohol use, drug use, and stealing. The subscale demonstrated good reliability across time (Cronbach's $\alpha=$ 0.73 at T1 and 0.77 at T2). Possible scores of self-reported risk taking range from 0 to 30 . In the current sample, the range was $1-11$ at $\mathrm{T} 1$ and $0-13$ at T2. There was no observable change in self-reported risk-taking behavior over time $(\mathrm{T} 1$ : mean $=5.14 ; \mathrm{SD}=2.87 ; \mathrm{T} 2$ : mean $=5.00 ; \mathrm{SD}=$ $3.38, t_{(21)}=0.26$, not significant). There were no differences based on participant sex. To examine how changes in risk taking covary with changes in neural activation, we created an index that represents the difference in risky behavior from $\mathrm{T} 1$ to $\mathrm{T} 2$ (i.e., $\mathrm{T} 2-\mathrm{T} 1$ raw difference score).

\section{fMRI paradigm}

To examine neural sensitivity to risk taking, participants completed the Balloon Analog Risk Task (BART; Lejuez et al., 2002; Euser et al., 2013). Behavioral performance on the BART has shown associations with actual risky behaviors, such as adolescent smoking, addiction, and drug use (Lejuez et al., 2003, 2007; Aklin et al., 2005), suggesting that this task is an ecologically valid measure of real-life risk taking. Furthermore, the BART is widely used in neuroimaging studies to examine neural responses to risk taking across different developmental ages (Rao et al., 2008; Chiu et al., 2012; Galván et al., 2013; Telzer et al., 2014).

On each trial of the task, participants are presented with a virtual red balloon. By pressing corresponding buttons, participants can choose either a risky option (i.e., pump the balloon), which results in bigger monetary rewards but a greater probability of getting no rewards (i.e., explosion of the balloon), or a safe option (i.e., cash out current rewards). For each successful pump without explosion, participants received 25 cents. However, if the balloon explodes before cashing out, participants received no payoff for that trial. As number of pumps increases during a trial, explosion probability increases exponentially. The explosion point of each balloon was drawn from a uniform distribution from 1 to 12 pumps. After each pump, the balloon image disappeared for a jittered interval of 1-3 s before the outcome was displayed, either a larger balloon or an exploded one. There was an interstimulus interval of variable (jittered) length ranging from 1 to $12 \mathrm{~s}$ (mean $=4 \mathrm{~s}$ ) after the end of each balloon trial (i.e., after explosion or cash-out). The payoff for each was accumulated, and participants received the total payoff at the end of the task. In addition to red balloons, participants were presented with white balloons, which were not associated with a reward or possible explosion. White balloons did not explode but inflated according to the same distribution as the red balloons and therefore were not associated with risk. The task was self-paced and was completed during one 9 min run.

\section{fMRI acquisition and analysis}

fMRI data acquisition. Imaging data were collected using a 3.0 tesla Siemens Trio MRI scanner. The BART consisted of T2*-weighted echoplanar images (EPIs; slice thickness $=4 \mathrm{~mm} ; 34$ slices; $\mathrm{TR}=2000 \mathrm{~ms}$; $\mathrm{TE}=$ $30 \mathrm{~ms}$; flip angle $=90^{\circ}$; matrix $=64 \times 64 ; \mathrm{FOV}=200 \mathrm{~mm}$; voxel size $=$ $3 \times 3 \times 4 \mathrm{~mm}^{3}$ ). A T2*-weighted, matched-bandwidth (MBW), highresolution, anatomical scan and a magnetization-prepared rapidacquisition gradient echo (MPRAGE) scan were acquired for registration purposes $(\mathrm{TR}=2.3 \mathrm{~ms} ; \mathrm{TE}=2.1 \mathrm{~ms} ; \mathrm{FOV}=256 \mathrm{~mm}$; matrix $=192 \times$ 192; sagittal plane; slice thickness $=1 \mathrm{~mm} ; 160$ slices). The orientation for the MBW and EPI scans was oblique axial to maximize brain coverage.

fMRI data preprocessing and analysis. Analyses were performed using Statistical Parametric Mapping (SPM8; Wellcome Department of Cognitive Neurology, Institute of Neurology, London, UK). Preprocessing for each participant's images included spatial realignment to correct for head motion (no participant exceeded $2 \mathrm{~mm}$ of maximum image-toimage motion in any direction). For each participant, the realigned functional data at $\mathrm{T} 1$ and $\mathrm{T} 2$ were coregistered to the corresponding $\mathrm{T} 1$ and T2 high resolution MPRAGE, which was then segmented into CSF, gray matter, and white matter. The normalization transformation matrix from the segmentation step was then applied to the functional and T2 structural images, thus transforming them into standard stereotactic space as defined by the Montreal Neurological Institute and the International Consortium for Brain Mapping. The normalized functional data were smoothed using a $8 \mathrm{~mm}$ Gaussian kernel, full-width-at-half maximum, to increase the signal-to-noise ratio. Each trial was convolved with the canonical hemodynamic response function. High-pass temporal filtering with a cutoff of $128 \mathrm{~s}$ was applied to remove low-frequency drift in the time series. Serial autocorrelations were estimated with a restricted maximum likelihood algorithm with an autoregressive model order of 1 .

Statistical analyses were performed using the general linear model (GLM) in SPM8. It is important to ensure the longitudinal changes in brain activation are specific to risk taking and not to changes in baseline. Thus, to control for any potential changes in global brain activation over time, we modeled the first-level models by concatenating neural activation at $\mathrm{T} 1$ and $\mathrm{T} 2$, such that the implicit baseline is averaged across the two time points. In each participant's fixed-effects analysis, a GLM was created with regressors of interest to separate different events: risk taking at $\mathrm{T} 1$ and $\mathrm{T} 2$ (i.e., pumps for red balloons), receipt of rewards at $\mathrm{T} 1$ and $\mathrm{T} 2$ (i.e., cash-outs), receipt of negative outcome at T1 and T2 (i.e., explosions), and control balloons at T1 and T2 (i.e., pumps on white balloons). Each event was modeled as the onset when the balloon appeared with a 
duration calculated as the response time when the participant chose to either pump or cash out. Following prior studies, for the risk-taking event, we analyzed pumps on balloons that did not explode (i.e., pumps on each balloon before cash-out), because pumps on the explosion trials were necessarily constrained (Lejuez et al., 2002). A parametric modulator was included as a control, which represented the pump number for each individual pump within a balloon. Pump number was mean centered within the individual. The parametric modulator served to control for differences across pumps within a balloon trial. Null events, consisting of the jittered intertrial intervals, were not explicitly modeled and therefore constituted an implicit baseline.

The individual-level contrast images were used in all group-level analyses, which were run using GLMflex. GLMflex uses partitioned error terms, corrects for variance-covariance inequality, removes outliers and sudden changes in brain activation, and analyzes all voxels that have data (http://mrtools.mgh.harvard.edu/index.php/ G LM_Flex). To examine longitudinal changes in neural reactivity during risk taking, we focused on the contrast comparing activity during risky decisions at $\mathrm{T} 1$ and $\mathrm{T} 2$ (T2 pumps minus $\mathrm{T} 1$ pumps for red balloons). To test how changes in neural reactivity covaried with changes in self-reported risk taking, we conducted whole-brain regression analyses, in which we examined how changes in risk taking (computed as the difference between T2 and T1 and entered as a regressor) related to the difference in neural activation during risky decisions from T2 to T1. Finally, to examine how functional coupling between the ventral striatum and cognitive control regions change over time, we conducted psychophysiological interaction (PPI) analyses. We used the VS as the seed region, because it is consistently related to reward seeking and risk-taking behavior (Delgado et al., 2000; Knutson et al., 2000). We used a functional ROI for the VS based on the region that showed significant activation at T1. PPI analyses were run using a generalized form of contextdependent PPI. Specifically, the automated generalized PPI (gPPI) toolbox in SPM (McLaren et al., 2012) was used to (1) extract the deconvolved times series from the ventral striatum ROI for each participant to create the physiological variables; (2) convolve each trial type with the canonical HRF, creating the psychological regressor; and (3) multiply the time series from the psychological regressors with the physiological variable to create the PPI term. This interaction term identified regions that covaried in a taskdependent manner with the VS. For each participant, one regressor representing the deconvolved BOLD signal was included alongside each psychological and PPI term for each event type to create a gPPI model.

The fMRI analyses included several covariates to control for learning effects within and across sessions. These covariates included change in behavioral performance on the BART across time, which represented the change in average number of pumps at T2 minus T1. In the fMRI analyses, we entered this behavioral change by treating it as a covariate. We did so because changes in average number of pumps lead to a different number of trials available at each time point. By controlling for this, we account for neural differences being driven by a different number of trials available at each time point. These covariates also included change in functional brain activation within the first session. To rule out the possibility that our results are driven by repetition effects, we defined regions that showed significant results in the longitudinal fMRI analyses. To do this, we first ran whole-brain analyses to identify brain regions that covaried across time. We then extracted activation from these regions and calculated the change in brain activation within the first session (i.e., brain activation in the second half of the task minus brain activation in the first half of the task) and controlled this change in the fMRI analyses. This ensured that any effects identified longitudinally were not being driven by repetition or learning effects.
To correct for multiple comparisons, we conducted a Monte Carlo simulation implemented using 3dClustSim in the software package AFNI (Analysis of Functional NeuroImages; http://afni.nimh.nih.gov/afni/). We used our group-level brain mask combined with the gray mask in SPM, therefore representing neural coverage in our sample that corresponded to gray matter. Results of the simulation indicated a voxelwise threshold of $p<0.005$ combined with a minimum cluster size of 34 voxels, corresponding to $p<0.05$, familywise error corrected. All statistical analyses reported are two-tailed. We used the MarsBaR toolbox within SPM to extract parameter estimates from significant clusters in the group-level analyses. Parameter estimates of signal intensity were extracted from the entire cluster of activation. For visualization purposes, statistical maps of all analyses were projected onto a T2 template. The scatter plots based on regression analyses are for illustration purposes only and do not represent independent analyses.

\section{Results}

\section{Behavioral results}

Following the example of previous studies (Lejuez et al., 2003), we assessed risk-taking behavior on the BART by examining the number of pumps before cash-outs, with a greater number of pumps before cash-outs indicating greater risk-taking behavior. Adolescents inflated 3.42 (SD between participants $=0.89$ ) pumps per balloon at $\mathrm{T} 1$, and 3.19 (SD between participants = $0.90)$ pumps per balloon at T2. No difference was observed in the average number of pumps between $\mathrm{T} 1$ and $\mathrm{T} 2\left(t_{(21)}=1.51, p=\right.$ $0.15)$. The SD for number of pumps within participants (i.e., within-person variability in pumps with higher scores representing more exploratory behavior) was 1.46 at T1 and 1.29 at T2, with no difference across the two time points $\left(t_{(21)}=1.61, p=\right.$ 0.12 ). Although we did not observe overall change in behavioral performance on the BART from $\mathrm{T} 1$ to $\mathrm{T} 2$, declines in selfreported risk-taking behavior covaried with declines in risk taking on the BART $\left(r_{(20)}=0.44, p=0.04\right.$; Fig. 1$)$, suggesting the task is ecologically valid and captures real changes in adolescents' risky behavior. 
Table 1. Brain activity during risk-taking behavior

\begin{tabular}{|c|c|c|c|c|c|c|}
\hline \multirow[b]{2}{*}{ Anatomical region } & \multirow{2}{*}{$\begin{array}{l}\text { Brodmann's } \\
\text { areas }\end{array}$} & \multicolumn{3}{|c|}{$\begin{array}{l}\text { Montreal Neurological } \\
\text { Institute coordinates }\end{array}$} & \multirow[b]{2}{*}{$t^{a}$} & \multirow[b]{2}{*}{$k^{b}$} \\
\hline & & $x$ & $y$ & $z$ & & \\
\hline \multicolumn{7}{|l|}{ Time 1} \\
\hline Left VLPFC & 10 & -27 & 59 & -8 & 5.58 & 142 \\
\hline Right VLPFC & 10 & 30 & 56 & 10 & 5.58 & 278 \\
\hline Left dorsolateral PFC & 9 & -33 & 41 & 28 & 7.09 & 233 \\
\hline Right dorsolateral PFC & $9 / 46$ & 33 & 38 & 31 & 5.84 & 357 \\
\hline Left VS & & -9 & 8 & -2 & 8.67 & 76 \\
\hline Right VS & & 15 & 11 & -8 & 7.94 & 110 \\
\hline Left insula & $13 / 45$ & -36 & 11 & 1 & 8.36 & 206 \\
\hline Right insula & $13 / 45$ & 33 & 20 & 4 & 8.31 & 149 \\
\hline $\mathrm{ACC}$ & $24 / 32$ & -6 & 8 & 28 & 6.99 & 237 \\
\hline \multicolumn{7}{|l|}{ Time 2} \\
\hline Left VLPFC & 10 & -33 & 59 & 4 & 3.32 & 54 \\
\hline Right VLPFC & 10 & 39 & 62 & 1 & 3.87 & 158 \\
\hline Left dorsolateral PFC & 9 & -42 & 47 & 28 & 5.83 & 143 \\
\hline Right dorsolateral PFC & $9 / 46$ & 33 & 50 & 25 & 8.76 & 270 \\
\hline Left VS & & -15 & 8 & -2 & 8.04 & 71 \\
\hline Right VS & & 15 & 8 & -2 & 7.14 & 136 \\
\hline Left insula & $13 / 45$ & -33 & 23 & 7 & 8.65 & 252 \\
\hline Right insula & $13 / 45$ & 33 & 23 & 1 & 8.64 & 203 \\
\hline $\mathrm{ACC}$ & $24 / 32$ & 3 & 20 & 22 & 7.43 & 169 \\
\hline
\end{tabular}

$a_{t \text { score at those coordinates (local maxima). }}$

${ }^{b}$ Number of voxels in each significant cluster.
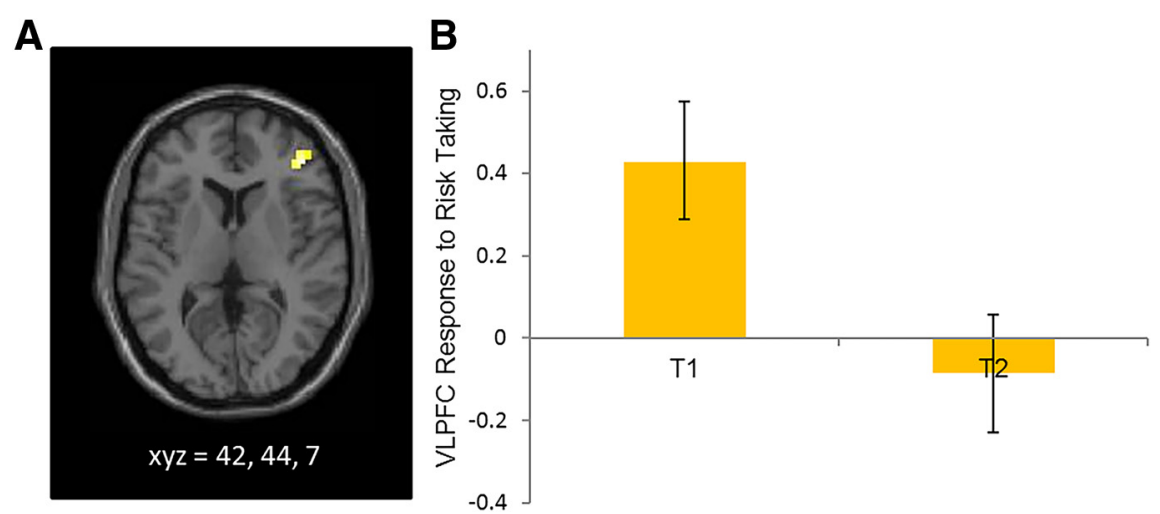

Figure 2. $\quad \boldsymbol{A}$, Longitudinal declines in VLPFC activation during risk taking from $T 1$ to $T 2 . \boldsymbol{B}, \mathrm{BOLD}$ response in the VLPFC during risk taking (means and SEM) relative to the implicit baseline at $\mathrm{T} 1$ and $\mathrm{T} 2$.

\section{fMRI results}

Neural responses to risk taking at $\mathrm{T} 1$ and $\mathrm{T} 2$

We first examined neural reactivity when adolescents made risky decisions (i.e., pumps) relative to the implicit baseline at T1 and T2. Whole-brain analyses showed heightened activation in both cognitive control and reward-related networks during risk taking at T1 and T2, including the VLPFC, dorsolateral PFC, VS, insula, and anterior cingulate cortex (Table 1).

\section{Longitudinal changes in neural responses to risk taking}

In whole-brain analyses, we examined neural reactivity when adolescents chose to make risky decisions at T2 compared with risky decisions at T1. Adolescents showed longitudinal decreases in activation in the right VLPFC when making risky decisions over time. This finding remained significant when controlling for within-session differences in functional activation in the VLPFC as well as between-session differences in behavioral performance $\left(t_{(19)}=4.45, p<0.001\right.$; Fig. $\left.2 A\right)$. For descriptive purposes, we extracted VLPFC activation at T1 and T2. Whereas adolescents showed heightened activation in the VLPFC during risk taking at
T1, the VLPFC did not evidence heightened activation at T2 (Fig. $2 B$ ). There were no other brain regions that showed longitudinal changes during risk taking.

We did not find evidence of longitudinal changes during the outcome phase (explosions or cash-outs). These results suggest that our effects are specific to changes in risk taking. To support that our findings are specific to risk taking, we examined longitudinal changes in brain activation during the white balloon condition (i.e., no monetary value was associated with pumping white balloons, which never exploded and therefore did not involve risk). Adolescents did not show statistically significant changes in brain activation across time during the pumping of white balloons in any brain region, providing further support that differences in VLPFC activation are specific to the risk-taking contrast.

Next, to eliminate the possibility that our findings are driven by learning effect, we modeled brain activation during the first half and second half of the BART at T1. There was no significant change in neural activation from the first half to the second half of the task at $p<0.005$. No significant change in neural activation was found even when we used a more liberal threshold of $p<$ 0.01 . There was also no significant change in behavioral performance from the first half to the second half of the task. These findings suggest that there are not significant changes in learning occurring across the task.

\section{Longitudinal relationships between brain and behavior}

We examined how individual differences in neurodevelopmental changes during risk taking were associated with changes in adolescents' self-reported risk-taking behavior. In whole-brain regression analyses, we regressed changes in self-reported risk taking on changes in neural reactivity during risk taking. Adolescents who showed greater declines in self-reported risk taking over time showed greater declines in neural reactivity in the VLPFC and VS. This finding remained significant when controlling for within-session differences in functional activation in the VLPFC and VS as well as between-session differences in behavioral performance (VLPFC: $t_{(16)}=5.11, p<0.001$; Fig. $3 A$; VS: $t_{(16)}=4.36, p<$ 0.001 ; Fig. $3 B$ ). Notably, the VLPFC region is the same region that showed longitudinal decreases in our first set of analyses. Indeed, when we localized the functional ROI for the VLPFC based on the previous analyses (i.e., the VLPFC cluster that showed significant changes from $\mathrm{T} 1$ to $\mathrm{T} 2$ ) and used this ROI to quantify its association with changes in self-reported risk taking, this area in the VLPFC was significantly associated with changes in self-reported risk taking $\left(r_{(20)}=0.52, p=0.01\right.$.

\section{PPI analysis}

Last, we conducted PPI analyses to examine longitudinal changes in functional coupling between the PFC and subcortical regions. We did not find evidence of average change in functional connectivity between the VS and PFC from T1 to T2. However, in wholebrain regression analyses, we did find that changes in neural coupling were associated with changes in self-reported risk taking. Specifically, adolescents who showed greater declines in risk taking over time showed greater decreases in coupling between the VS and MPFC from T1 to T2. This finding remained significant 
when controlling for within-session differences in the MPFC-VS coupling and between-session differences in behavioral performance $\left(t_{(18)}=4.74, p<0.001\right.$; Fig. 4). Thus, the more adolescents changed toward greater negative coupling between these two regions over time, the less they engaged in risky behavior.

To fully interpret the direction of the change in neural coupling, we ran PPI analyses at T1 with self-reported risk taking at $\mathrm{T} 1$. We found a positive correlation between risk taking and MPFC-VS coupling $\left(t_{(20)}=3.87, p<0.001\right)$. As shown in Figure 5, positive coupling between the MPFC and VS was associated with greater T1 risk taking (Fig. 5, top right quadrant), whereas negative coupling between the MPFC and VS was associated with less T1 risk taking (Fig. 5, bottom left quadrant). Notably, this activation is in the identical region identified in the longitudinal analysis described above. Therefore, the MPFC-VS coupling plays a similar role at T1, and, together with our longitudinal effects, suggests that as adolescents' MPFCVS neural connectivity becomes more negatively coupled, they show greater declines in risk taking.

\section{Correlations between neural} activations

Finally, we examined the association between changes in VLPFC activation, changes in VS activation, and changes in MPFC-VS connectivity. To do this, we extracted parameter estimates of signal intensity from each region identified above and ran correlation analyses in SPSS. Changes in VLPFC activity are positively correlated with changes in MPFC-VS connectivity $\left(r_{(20)}=0.60, p=0.003\right)$, indicating that adolescents who show longitudinal declines in neural coupling also show longitudinal decreases in VLPFC activation. Similarly, changes in VLPFC activity are positively related to changes in VS activity $\left(r_{(20)}=0.72\right.$, $p<0.001$ ), and changes in VS activity are positively associated with changes in MPFC-VS connectivity $\left(r_{(20)}=0.48, p=0.03\right)$.

\section{Discussion}

Adolescence is a time of dramatic brain development, accompanied by changes in risk-taking behavior. In the current study, we used a longitudinal approach to examine the relationships between changes in brain and changes in risktaking behavior. Our findings provide novel evidence demonstrating the disparate roles of the VLPFC and MPFC in modulating declines in adolescent risk taking. While longitudinal declines in
A
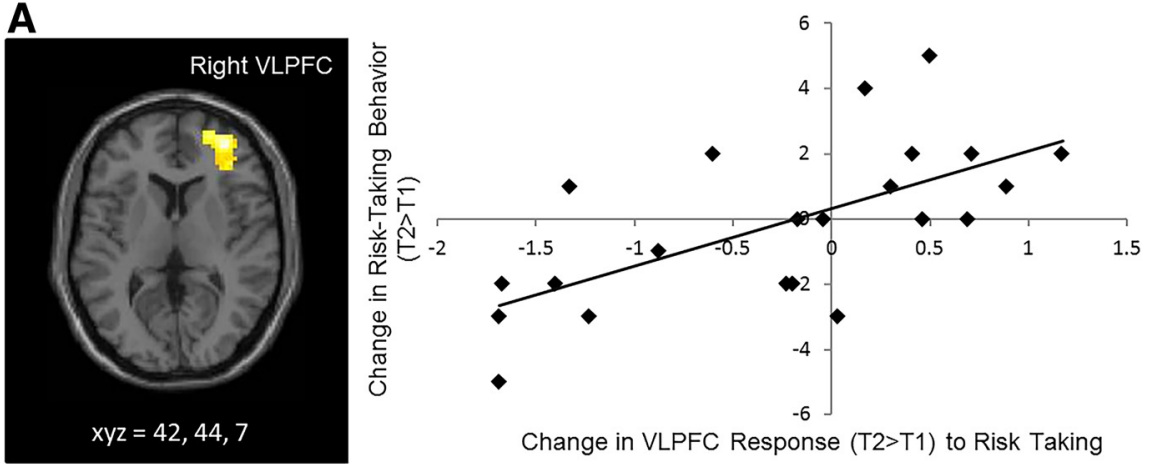

Change in VLPFC Response (T2>T1) to Risk Taking

B
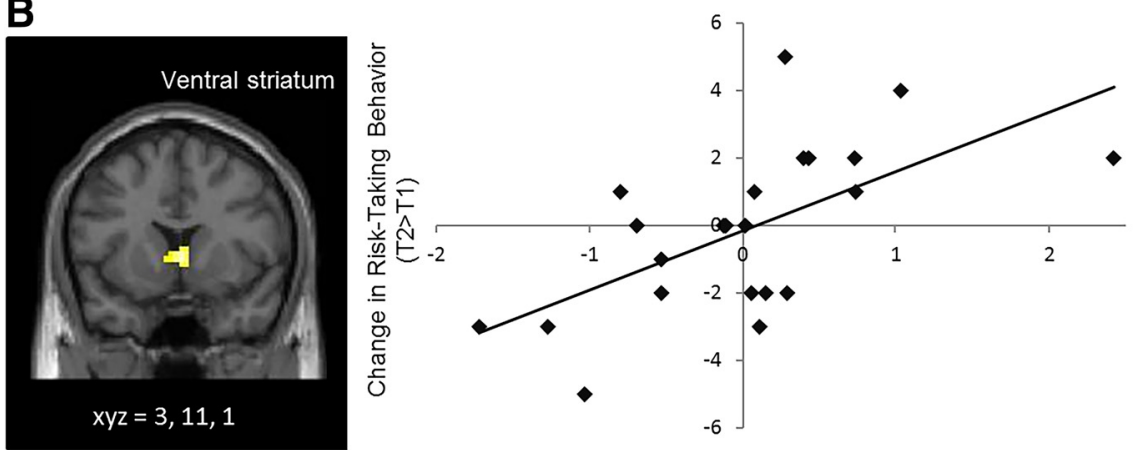

Change in VS Response (T2>T1) to Risk Taking

Figure 3. $\quad \boldsymbol{A}, \boldsymbol{B}$, Longitudinal changes in (A) VLPFC and (B) VS during risk taking correlate with longitudinal changes in selfreported risk-taking behavior. The scatterplots are not independent analyses and are presented for illustration purposes.
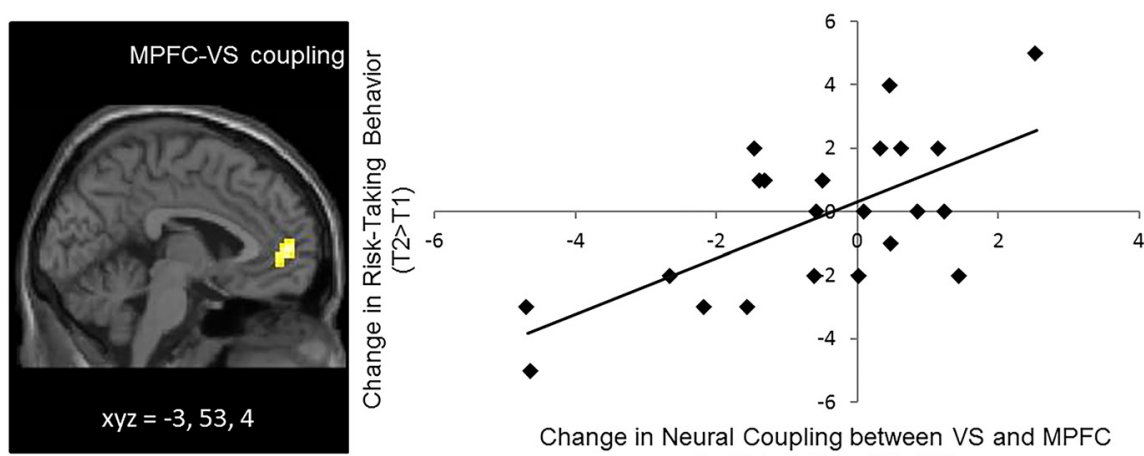

Change in Neural Coupling between VS and MPFC to Risk Taking (T2>T1)

Figure 4. Longitudinal changes in neural coupling between VS and MPFC correlate with longitudinal changes in self-reported risk-taking behavior. The scatterplot is not an independent analysis and is presented for illustration purposes.
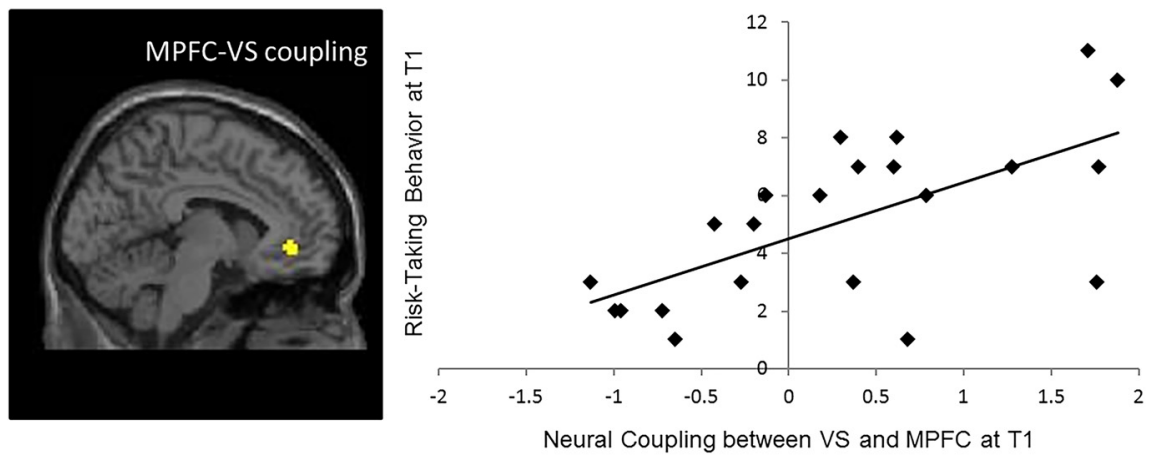

Figure 5. Neural coupling between VS and MPFC at T1 correlate with risk-taking behavior at T1. 
VLPFC activation were related to declines in risk taking over time, longitudinal changes in the MPFC were associated with declines in risk taking via decreased connectivity with the VS.

We found longitudinal declines in VLPFC activation during adolescent risk taking, with such declines associated with declines in self-reported risk-taking behavior. Prior research suggests that the VLPFC is involved in cognitive control and decision making under uncertainty (Levy and Wagner, 2011) as well as goaldirected inhibitory control, including the braking of motor responses (Wessel et al., 2013). However, divergent evidence has emerged in terms of the function of greater or lower PFC activity (Crone and Dahl, 2012). In the current study, we found longitudinal declines in VLPFC activation during risk taking, which were associated with declines in real-life risk taking, suggesting that a greater decrease in VLPFC activation over time may serve a protective function. Among lower risk takers, less VLPFC activation may be indicative of reduced need for control. In more riskprone individuals, greater VLPFC activation may be recruited to regulate the heightened VS response by engaging MPFC-VS coupling. Indeed, increases in VLPFC activation were associated with increases in VS activation as well as increases in MPFC-VS connectivity. A decline in VLPFC activation may also be indicative of pruning occurring during adolescence based on experience, resulting in less VLPFC activation over time.

The MPFC demonstrated a different neural pattern during risk taking. Specifically, changes in functional connectivity between the MPFC and VS were associated with changes in adolescent risk taking: adolescents who showed increased positive coupling exhibited greater risk-taking behavior over time, whereas those who showed increased negative coupling exhibited less risk-taking behavior. This is consistent with recent crosssectional work in human and rodent youth, which has shown that from childhood to adolescence, individuals demonstrate a developmental switch from positive to negative coupling between the MPFC and subcortical regions with negative coupling related to better psychological functioning (Perlman and Pelphrey, 2011; Gee et al., 2013). Our findings suggest that adolescents whose MPFC increases in "top-down" regulation of the ventral striatum show decreases in their risky behavior in real life.

In addition to the PFC, we found that declines in VS reactivity were associated with declines in self-reported risk taking. Heightened VS activation has consistently been associated with greater reward-seeking and risk-taking behavior and shows greater reactivity in adolescents than children or adults (Ernst et al., 2005; Galvan et al., 2006). Thus, adolescents who demonstrated marked declines in real-life risk taking may show declines in VS activity due to reduced reward sensitivity over time.

On average, we did not find changes in adolescents' self-report risk taking. This could be due to a lack of power given the small sample size. On the other hand, this may reflect the fact that although adolescence is a time of changes in risk-taking behavior, the average change may not be observed at the group level. Instead, change may emerge at the individual level. That is, some adolescents may show increases in their risk-taking behavior while other adolescents may show decreases. Such variability appears as no change when examining group-level effects. It may be also the case for adolescents' neural development; while some adolescents may show increases in MPFC-VS connectivity, other adolescents may show decreases. Because not all adolescents will show change at the same rate or at the same time due to variability in brain development (Kraemer et al., 2000), brain-behavior relations may be the best way to capture developmental changes during adolescence. The key goal of the current study was to examine whether the individual differences in neural changes are meaningful in terms of their association with individual differences in risk-taking trajectories in real life. Indeed, we find that individual changes in VLPFC, VS, and MPFC-VS coupling are associated with changes in risk-taking behavior, suggesting that the changes over 1.5 years are informative and meaningful at the individual level. Future research is needed to examine neural changes and brain-behavior relations across more than two time points to gain a deeper understanding of developmental trajectories. Finally, we controlled for behavioral differences on the BART in our fMRI analyses to rule out the possibility that the association between changes in brain activation and changes in self-reported risk taking are driven by changes in behavioral performance on the task. Although this approach is more conservative, it may preclude our ability to find an association between changes in behavioral performance and changes in brain activation.

In conclusion, this longitudinal fMRI study provides a new perspective on the role of the PFC underlying changes in adolescent risk taking. Importantly, our findings provide novel evidence that disparate roles of the VLPFC and MPFC underlie changes in adolescent risk taking. While longitudinal declines in the VLPFC activity are directly associated with declines in risk taking, changes in the MPFC relate to declines in risk taking via greater negative connectivity with the VS over time. Future research should explore environmental factors that enhance the functions of different PFC regions as a way to protect adolescents from heightened risk-taking behavior.

\section{References}

Achenbach TM (1991) Integrative guide for the 1991 CBCL/4-18, YSR, and TRF profiles. Burlington, VT: University of Vermont Department of Psychiatry.

Aklin WM, Lejuez CW, Zvolensky MJ, Kahler CW, Gwadz M (2005) Evaluation of behavioral measures of risk taking propensity with inner city adolescents. Behav Res Ther 43:215-228. CrossRef Medline

Booth JR, Burman DD, Meyer JR, Lei Z, Trommer BL, Davenport ND, Li W, Parrish TB, Gitelman DR, Mesulam MM (2003) Neural development of selective attention and response inhibition. Neuroimage 20:737-751. CrossRef Medline

Bunge SA, Dudukovic NM, Thomason ME, Vaidya CJ, Gabrieli JD (2002) Immature frontal lobe contributions to cognitive control in children: evidence from fMRI. Neuron 33:301-311. CrossRef Medline

Casey BJ, Getz S, Galvan A (2008) The adolescent brain. Dev Rev 28:62-77. CrossRef Medline

Chiu CY, Tlustos SJ, Walz NC, Holland SK, Eliassen JC, Bernard L, Wade SL (2012) Neural correlates of risky decision making in adolescents with and without traumatic brain injury using the balloon analog risk task. Dev Neuropsychol 37:176-183. CrossRef Medline

Crone EA, Dahl RE (2012) Understanding adolescence as a period of socialaffective engagement and goal flexibility. Nat Rev Neurosci 13:636-650. CrossRef Medline

Delgado MR, Nystrom LE, Fissell C, Noll DC, Fiez JA (2000) Tracking the hemodynamic responses to reward and punishment in the striatum. J Neurophysiol 84:3072-3077. Medline

Durston S, Davidson MC, Tottenham N, Galvan A, Spicer J, Fossella JA, Casey BJ (2006) A shift from diffuse to focal cortical activity with development. Dev Sci 9:1-8. CrossRef Medline

Ernst M, Nelson EE, Jazbec S, McClure EB, Monk CS, Leibenluft E, Blair J, Pine DS (2005) Amygdala and nucleus accumbens in responses to receipt and omission of gains in adults and adolescents. Neuroimage 25: 1279-1291. CrossRef Medline

Ernst M, Pine DS, Hardin M (2006) Triadic model of the neurobiology of motivated behavior in adolescence. Psychol Med 36:299-312. Medline

Euser AS, Evans BE, Greaves-Lord K, Huizink AC, Franken IH (2013) Parental rearing behavior prospectively predicts adolescents' risky decision-making and feedback-related electrical brain activity. Dev Sci 16:409-427. CrossRef Medline 
Galvan A, Hare TA, Parra CE, Penn J, Voss H, Glover G, Casey BJ (2006) Earlier development of the accumbens relative to orbitofrontal cortex might underlie risk-taking behavior in adolescents. J Neurosci 26:68856892. CrossRef Medline

Galván A, Schonberg T, Mumford J, Kohno M, Poldrack RA, London ED (2013) Greater risk sensitivity of dorsolateral prefrontal cortex in young smokers than in nonsmokers. Psychopharmacology (Berl) 229:345-355. CrossRef Medline

Gee DG, Humphreys KL, Flannery J, Goff B, Telzer EH, Shapiro M, Hare TA, Bookheimer SY, Tottenham N (2013) A developmental shift from positive to negative connectivity in human amygdala-prefrontal circuitry. J Neurosci 33:4584-4593. CrossRef Medline

Knutson B, Westdorp A, Kaiser E, Hommer D (2000) fMRI visualization of brain activity during a monetary incentive delay task. Neuroimage 12: 20-27. CrossRef Medline

Kraemer HC, Yesavage JA, Taylor JL, Kupfer D (2000) How can we learn about developmental processes from cross-sectional studies, or can we? Am J Psychiatry 157:163-171. CrossRef Medline

Laviola G, Macrì S, Morley-Fletcher S, Adriani W (2003) Risk-taking behavior in adolescent mice: psychobiological determinants and early epigenetic influence. Neurosci Biobehav Rev 27:19-31. CrossRef Medline

Lejuez CW, Read JP, Kahler CW, Richards JB, Ramsey SE, Stuart GL, Strong DR, Brown RA (2002) Evaluation of a behavioral measure of risk taking: The balloon analogue risk task (BART). J Exp Psychol Appl 8:75-84. CrossRef Medline

Lejuez CW, Aklin WM, Zvolensky MJ, Pedulla CM (2003) Evaluation of the balloon analogue risk task (BART) as a predictor of adolescent real-world risk-taking behaviors. J Adolesc 26:475-479. CrossRef Medline

Lejuez CW, Aklin W, Daughters S, Zvolensky M, Kahler C, Gwadz M (2007) Reliability and validity of the youth version of the balloon analogue risk task (BART-Y) in the assessment of risk-taking behavior among innercity adolescents. J Clin Child Adolesc Psychol 36:106-111. Medline

Levy BJ, Wagner AD (2011) Cognitive control and right ventrolateral prefrontal cortex: reflexive reorienting, motor inhibition, and action updating. Ann NY Acad Sci 1224:40-62. CrossRef Medline

Marsh R, Zhu H, Schultz RT, Quackenbush G, Royal J, Skudlarski P, Peterson BS (2006) A developmental fMRI study of self-regulatory control. Hum Brain Mapp 27:848-863. CrossRef Medline

McLaren DG, Ries ML, Xu G, Johnson SC (2012) A generalized form of context-dependent psychophysiological interactions (gPPI): A compari- son to standard approaches. Neuroimage 61:1277-1286. CrossRef Medline

Perlman SB, Pelphrey KA (2011) Developing connections for affective regulation: age-related changes in emotional brain connectivity. J Exp Child Psychol 108:607-620. CrossRef Medline

Pfeifer JH, Masten CL, Moore WE 3rd, Oswald TM, Mazziotta JC, Iacoboni M, Dapretto M (2011) Entering adolescence: resistance to peer influence, risky behavior, and neural changes in emotion reactivity. Neuron 69:1029-1036. CrossRef Medline

Rao H, Korczykowski M, Pluta J, Hoang A, Detre JA (2008) Neural correlates of voluntary and involuntary risk taking in the human brain: an fMRI study of the Balloon Analog Risk Task (BART). Neuroimage 42: 902-910. CrossRef Medline

Rubia K, Smith AB, Taylor E, Brammer M (2007) Linear age-correlated functional development of right inferior frontostriato-cerebellar networks during response inhibition and anterior cingulate during errorrelated processes. Hum Brain Mapp 28:1163-1177. CrossRef Medline

Spear LP (2000) The adolescent brain and age-related behavioral manifestations. Neurosci Biobehav Rev 24:417-463. CrossRef Medline

Steinberg L (2008) A social neuroscience perspective on adolescent risktaking. Dev Rev 28:78-106. CrossRef Medline

Telzer EH, Fuligni AJ, Lieberman MD, Galván A (2014) Neural sensitivity to eudaimonic and hedonic rewards differentially predict adolescent depressive symptoms over time. Proc Natl Acad Sci U S A 111:6600-6605. CrossRef Medline

van den Bulk BG, Koolschijn PC, Meens PH, van Lang ND, van der Wee NJ, Rombouts SA, Vermeiren RR, Crone EA (2013) How stable is activation in the amygdala and prefrontal cortex in adolescence? A study of emotional face processing across three measurements. Dev Cogn Neurosci 4:65-76. CrossRef Medline

van Duijvenvoorde AC, Op de Macks ZA, Overgaauw S, Gunther Moor B, Dahl RE, Crone EA (2014) A cross-sectional and longitudinal analysis of neural responses to rewards: effects of age, pubertal stage, and frontostriatal connectivity. Brain Cogn 89:3-14. CrossRef Medline

Velanova K, Wheeler ME, Luna B (2009) The maturation of task set-related activation supports late developmental improvements in inhibitory control. J Neurosci 29:12558-12567. CrossRef Medline

Wessel JR, Conner CR, Aron AR, Tandon N (2013) Chronometric electrical stimulation of right inferior frontal cortex increases motor braking. J Neurosci 33:19611-19619. CrossRef Medline 